RAD Conference Proceedings, vol. 3, pp. 25-31, 2018

ISSN 2466-4626 (online) | DOI: 10.21175/RadProc.2018.06

www.rad-proceedings.org

\title{
THE USE OF THE FUNDAMENTAL PARAMETERS METHOD TO CALCULATE THE CONCENTRATIONS OF ELEMENTS IN ENVIRONMENTAL SAMPLES USING XRF PORTABLE SYSTEMS
}

\author{
Alketa Sinanaj*, Blerina Papajani \\ Faculty of Natural Science, Aleksander Xhuvani University, Elbasan, Albania
}

\begin{abstract}
The X-ray fluorescence method is known as a laboratory method and one of the few atomic spectroscopy methods used in many fields. The process of emission of the characteristic $X$-rays is called "X-ray fluorescence" $(X R F)$. The objectives of this research study are optimization of the XRF portable system geometry and the use of the fundamental parameters method to calculate the concentrations of elements in environmental samples using portable systems. Since this method is quick in getting results and does not destroy the sample, it is widely used both in research and in the analysis of industrial products for materials in the field of mineralogy, geology, environmental analysis of water, air, etc. Various experiments have led to the optimization of the geometry system, as the distances' minimization, angles optimization, and the assessment of the beam spot in the position of the sample. Most of the data collected are used as initial data in the ADMCA program that uses fundamental parameters (FP-XRF) for calculating the concentrations of elements in environmental samples. Errors in calculating the concentrations of the sediment and soil samples when we introduce the approximate content of light elements can be eligible for geochemical studies. During the research, several areas that can be used in the future to improve the results are identified.
\end{abstract}

Key words: XRF (X-Ray Fluorescence), WDXRF (Wavelength Dispersive X-Ray Fluorescence), EDXRF (Energy Dispersive X-Ray Fluorescence), FP (Fundamental Parameters)

\section{MECHANISM OF ORIGIN OF X-RAY FLUORESCENCE}

The mechanism of the production of the characteristic X-radiation includes the ionization of atoms in the interior electronic layers and the filling of vacancies created by the electrons crossing to the layers above, as a result of which a photon of X-radiation is emitted [1], [2].

When the method of X-ray fluorescence (XRF) is used, the ionization of atoms in the sample is mainly performed using $\mathrm{X}$ or $\mathrm{\gamma}$-radiation which ionizes the atom due to the photoelectric effect [3], [4]. During the radiation of the atom with particles as photons or electrons with sufficient energy, electrons will be ejected, creating vacancies (ionization) at a certain electrons level. In this way, an excited atom is an unstable system. It can return to the ground state by filling the vacancy created with one of the electrons located at one of the highest energy levels [9]. Whenever an electron moves from a higher energy level $j$ to a lower energy level $i$, the atom loses energy in the form of electromagnetic radiation that is named characteristic radiation or fluorescence. The principle of conservation of energy states that every electron transition from level $\mathrm{j}$ to level $\mathrm{i}$ will be accompanied by the emission of a photon whose energy is:

$$
(h v)_{j, i}=E_{j}-E_{i}
$$

Photoelectric absorption is not the only process that leads to the weakening of the primary radiation during its spread in the sample. An important process is that of diffusion. There are two main types of diffusion, named respectively Compton and Reyleigh [5].

\section{EQUATION OF X-RAY FLUORESCENCE}

The realization of a quantitative analysis requires a mathematical relation between the analytical parameter, which in this case is the radiation intensity of a line spectrum of fluorescence- $\mathrm{X}$ that corresponds to an element and its concentration. The determination of such a relationship has been the object of study of many authors, such as Blokhin, Sherman, Dan, Kote, etc. The determination of the fluorescence equation is conducted under certain geometry and the excitation of the sample, which, however, does not affect the essence of the problem [5].

The Fundamental Parameters method is based on a physical theory that enables the calculation of the intensity of fluorescent radiation which originates from a known sample composition. Equations usually

*alketasinanaj@hotmail.com 
A. Sinanaj, B. Papajani, The use of the fundamental parameters method..., RAD Conf. Proc., vol. 3, 2018, 25-31

consider primary and secondary fluorescence. Equations to calculate the intensity of the fluorescent radiation were proposed soon after the introduction of XRF spectrometers in the early 1950s. The equation of $\mathrm{X}$-ray fluorescence that takes into consideration the absorption of the primary and secondary rays by all elements is [6], [7], [8]:

$$
I_{i}=P_{i} W_{i} \int_{\lambda_{\min }}^{\lambda_{\text {edge }}} \frac{J(\lambda) \mu_{i}(\lambda)}{\sum_{j=1}^{n} W_{j} \mu_{j}(\lambda)+G \sum_{j=1}^{n} W_{j} \mu_{j}\left(\lambda_{i}\right)} d \lambda(2)
$$

$\mathrm{I}^{-}$is the intensity of the $\mathrm{K}_{\alpha}$ line of fluorescence radiation of "element $i$ ". $J(\lambda)$ - is the X-ray spectrum. $\mathrm{P}_{\mathrm{i}}$ - This factor is the effectiveness of excitementdetecting element and depends on the fundamental parameters that affect the excitation and detection of the analytical line of "element $i$ ". $W_{i}$ - is the concentration of the "element $i$ ". $G$ - is the geometric factor which depends on the geometry of the system (surfaces, distances, angles) and for certain geometric conditions it is constant. $\mu_{j}(\lambda)$ - is the absorption coefficient for element " $\mathrm{j}$ " for primary photons. $\mu_{j}\left(\lambda_{i}\right)-$ the absorption coefficient for element $\mathrm{j}$ for the characteristic wavelength $\lambda_{\mathrm{i}}$ of the analyte "i".

The Fundamental Parameters method it is intended to calculate the concentration of an element by iteratively solving the preceding equation. It is necessary to recognize the precise distribution of the primary radiation, the excitation geometry data, the features of the tube and detector, fundamental parameters of excitation and detection of the element, as well as the approximate composition of the sample.

\section{DESCRIPTION OF THE PORTABLE SYSTEM'S X-RAY FLUORESCENCE}

The portable spectrometer X-ray fluorescence with Energy Dispersion (EDXRF) consists of:

$>\quad X$-ray detector - The detector used in our system is a Si-PIN photodiode with a area of $7 \mathrm{~mm}^{2}$ and thickness of $300 \mu \mathrm{m}$ (model XR-1OOCR produced by AMPTEC INC., USA).

$>$ Electronic Devices of the Supply and Acquiring PX4 Spectrum (spectra processing programs and calculation of concentrations) - A PX4 electronic device named 'digital impulse processor' replaces some of the chain components of the signal processing such as sources of supply, the amplifier, the multi-channel analyzer, the logical circuit, etc. PX4 generates all voltages needed for the detector, its thermoelectric cooling, and various supplies of the preamplifier and its schemes. A program named ADMCA (Software ADMCA PX4) installed on a computer allows communication with the $\mathrm{PX}_{4}$, the control and regulation of all measurement parameters, the spectra manipulation of data storage, and the processing of spectra and the calculation of the concentration using the Fundamental Parameters method. ADMCA programs that serves to control the working parameters of the system, obtaining XRF spectra and their manipulation was put into operation and was used to measure a number of standard samples of soils and sediments, which were used to calculate the concentrations of elements with the fundamental parameter method (FP-XRF)

$>$ The Primary Radiation Source - The primary radiation source which is used in the portable system is the 'Mini-X' X-ray tube (Amptek INC., MA, USA).

$>$ Geometric Device for the Placement of the Source, Sample, and Detector - Both the 'Mini X' tube and the detector were mounted on a metal plate with a $90^{\circ}$ angle, which provides a lateral excitation geometry angle of $45^{\circ}$ directly of the placement of source-sample and sample-detector (Figure 1). The window where the sample is placed (measurement window) has the shape of a circle with a $30-\mathrm{mm}$ diameter and is mounted on the same board so that it can be transposed or rotated. This enables us to optimize the distance and angles of excitation of the sample.

Before the detector is placed, a pure-aluminum collimator with $3-\mathrm{mm}$ openings, which are slightly larger than the dimensions of the detector diode $(2.4 \mathrm{x}$ $2.8 \mathrm{~mm}$ ), is installed. This collimator is also used to prevent it from falling directly on the detector of highenergy radiation. A similar collimator, with a diameter of $1.5 \mathrm{~mm}$, is used when the device operated with a high-intensity primary beam.

A bronze collimator coated with aluminum with a diameter of $1 \mathrm{~mm}$ is located at the outlet of the Mini X' tube. Its holder allows setting filters when you need to use them.

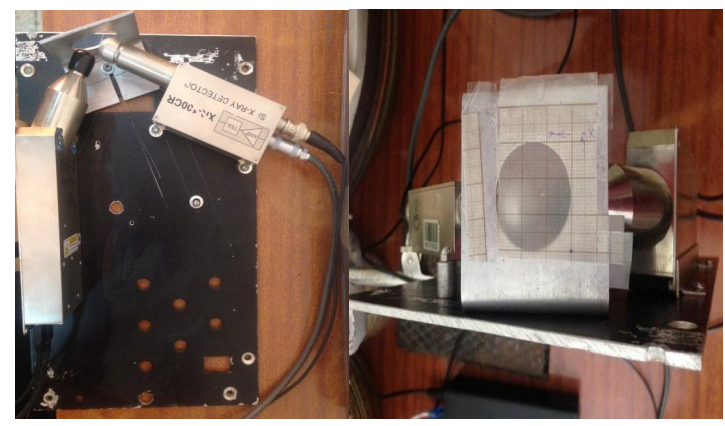

Figure 1. Photo of the geometric equipment scheme of the portable system (sideward, above)

The fluorescence equation indicates that the intensity of the measure depends largely on the geometry of the system (distances and angles). For the optimization of geometric equipment, we conducted some experiments related to placing the sample in the position with maximum intensity, finding the most appropriate angle and, finally, finding and assessing the distribution of the beam intensity of primary radiation in the optimum position of the sample. 
A. Sinanaj, B. Papajani, The use of the fundamental parameters method..., RAD Conf. Proc., vol. 3, 2018, 25-31

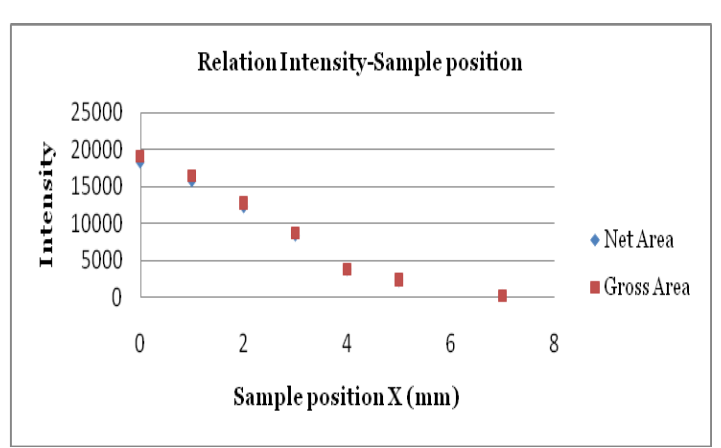

Figure 2. Dependency of the intensity from the sample position $\mathrm{X}(\mathrm{mm})$

Measurements show that the measured intensity is declining in relation to the relocation of the sample from the initial position. Thus, the closest possible position with the tube and detector was selected as the optimal placement position of the sample. In this position, the tube-sample and sample-detector distances are respectively 22 and $10 \mathrm{~mm}$. It is known that the effectiveness of the detection excitement is not constant within the measuring of the window surface. This happens as a result of several factors, such as the expansion of the primary radiation beam, change of the rigid angles under which both the source and the detector are viewed from any point, etc. Both the tube 'Mini X' and the detector were mounted on a metal plate with $90^{\circ}$ angle, which provides a lateral excitation geometry angles $45^{\circ}$ directly of the placement of source -sample and sample -detector. In order to evaluate the change in the effectiveness of the excitement-detection, an experiment was conducted consisting of measuring the intensity of the $\mathrm{Pb}-\mathrm{Sn} \mathrm{Ka}$ issued by a small piece of $\mathrm{Pb}-\mathrm{Sn}(1 \times 1 \mathrm{~mm})$, located in different positions in the window of measurement.

The results of the measurements performed along the two axes of the window are shown in Figure 3. These results indicate that the primary radiation blot on the sample position has the form of a circle with a diameter of approximately $6 \mathrm{~mm}$, despite the detector collimators. Similar results were acquired for both the $1.5-\mathrm{mm}$ and $3-\mathrm{mm}$ collimators. More than $90 \%$ of the information comes from these areas and the intensity varies more than 10 times from the center to the periphery of this area. Setting filters in the exit tube distorts the blot in a way due to the radiation diffusion. In the presence of $1 \mathrm{~mm}$ thick $\mathrm{Al}$, the blot has the shape of an ellipse with diameters of 8 and $6 \mathrm{~mm}$. It also turns out that window borders do not contribute to the measurements of intensity.

It should be noted that the recognition of this fact is especially important when analyzing samples with heterogeneous composition, while during normal procedure of analyzing the samples homogenized before measuring, the variation is not a problem because it is the same both in the measurement of the standards as well as in the measurement of the samples. Other important parameters of the geometric device are average decline angles and the X-radiation output from the sample. These parameters are important because their values are a part of the equation for calculating the concentrations of elements.
Finding the optimal placement of the distance sampling was performed by moving the sample holder for over half of the angle between the tube and detector and performing measurements at each of the positions. The position closer to the detector and the source (as close as the geometric dimensions allow) was taken as the 'o' position. The sample in the study was a slab of copper $(\mathrm{Cu})$. X-rays were emitted directly from the tube without using any filters. The excitation conditions are: $15 \mathrm{kV}$ of high voltage, $5.5 \mu \mathrm{A}$ of current, and a time of the intensity measurement of $10 \mathrm{sec}$. Three measurements were performed in each position.
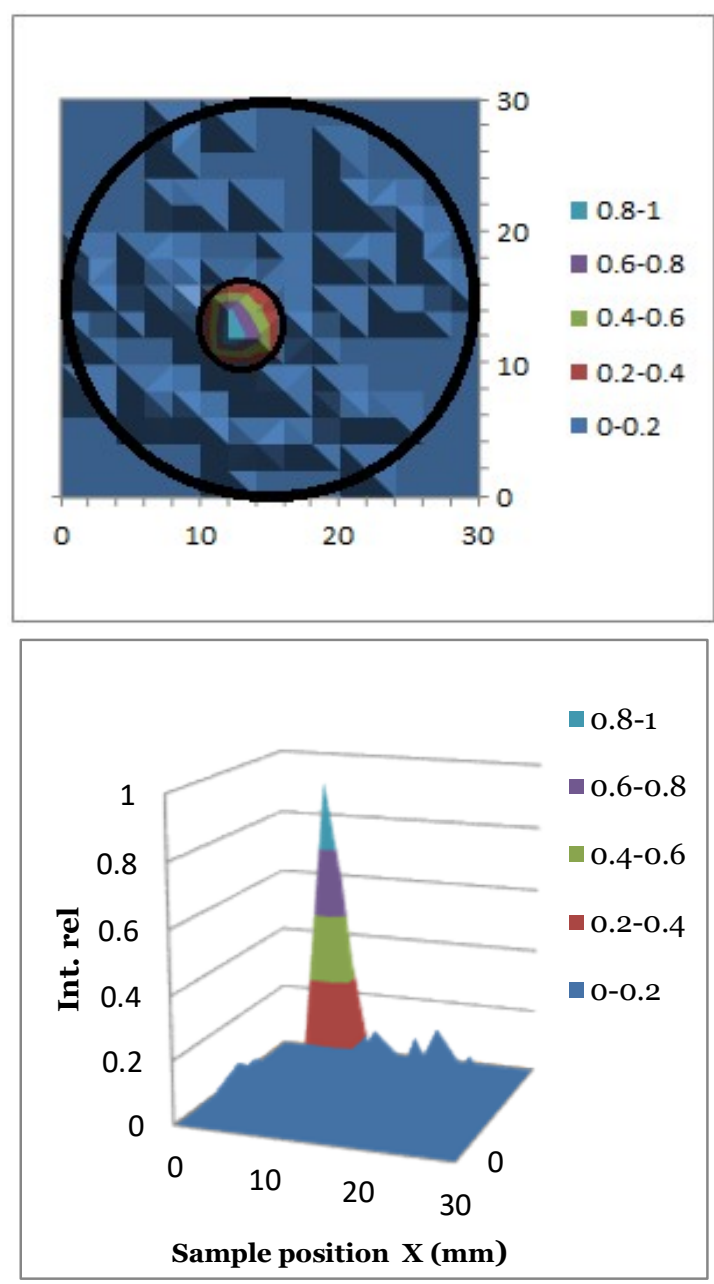

Figure 3. The shape, dimensions of the blot and the intensity change for collimator $3 \mathrm{~mm}$ 
A. Sinanaj, B. Papajani, The use of the fundamental parameters method..., RAD Conf. Proc., vol. 3, 2018, 25-31
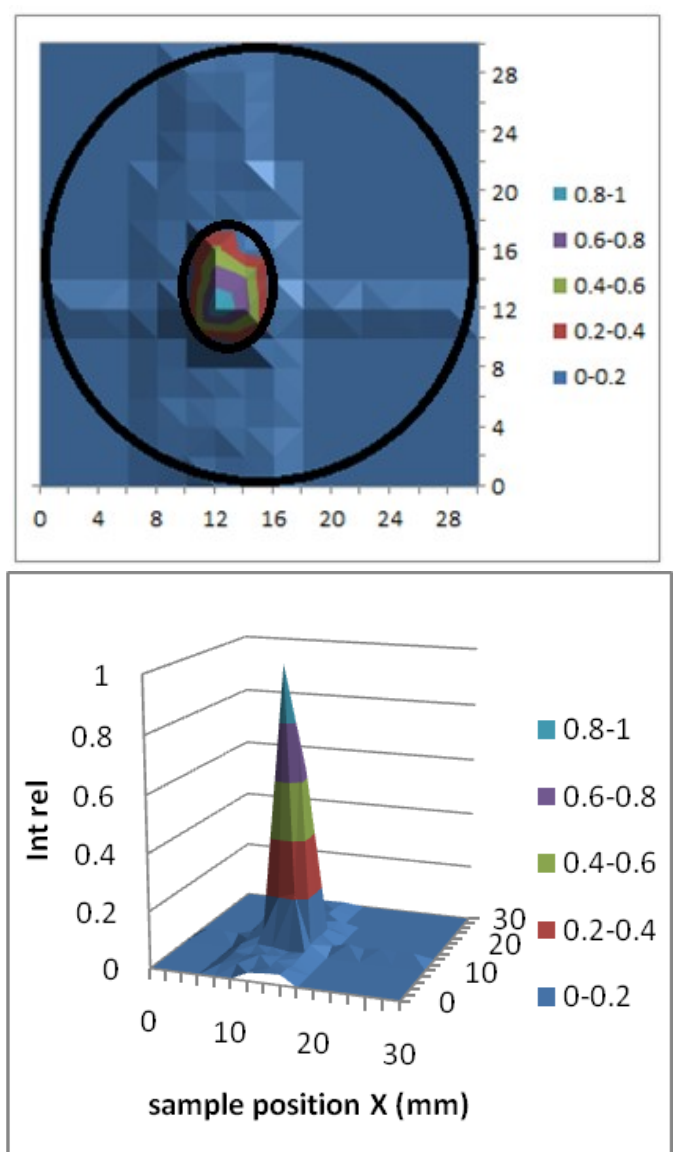

Figure 4. The shape, dimensions of the blot and the intensity change with $1 \mathrm{~mm} \mathrm{Al}$ filter and the 3-mm collimator

The third experiment is related to the impact of the fall/drop angle of the primary beam on the sample with the measured intensity. Having no opportunity to change the control of the correct angle, it sufficed to perform measurements in three extreme cases - a small angle of the fall (sample roughly parallel to the tube), angle of fall of $45^{\circ}$, defined by the geometry, and an angle of approximately $90^{\circ}$ (the sample approximately perpendicular to the tube). As in the case of the first experiment, it was measured in the same terms as the intensity of the radiation of a copper plate $(\mathrm{Cu})$. The results are presented in Figure 5.

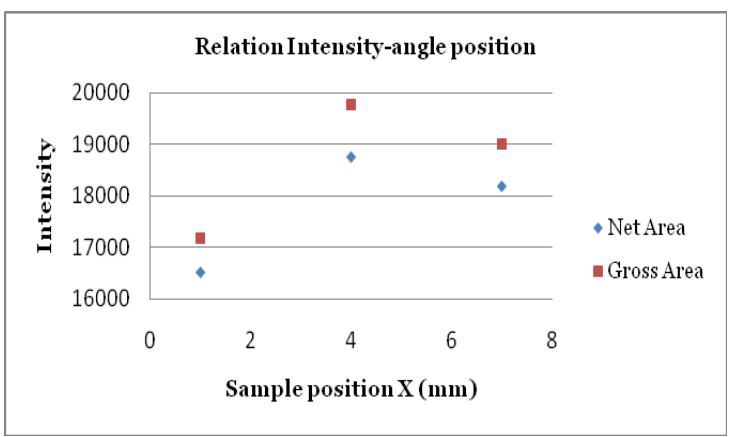

Figure 5. Dependency of the intensity of the radiation of the $\mathrm{Cu}$ $\mathrm{Ka}$ lines from the angular position of the sample in the window
We note that the intensity values change less than $10 \%$ depending on the angle and that they are greater for fall approximately to the zero angle when the projection of beam radiation on the sample is at its maximum. As the normal sample position, we will choose the angle drop of $45^{\circ}$, which allows a smaller tube-sample-detector distance and a lower intensity of diffused radiation.

\section{Calibration}

The use of the Fundamental Parameters method for calculating the concentrations of elements in a sample tends to resolve the general equation of fluorescence based on:

$>$ The fluorescence intensities of the elements of the sample (preferably all component elements, $\Sigma$ ci $=$ 100)

$>$ Energy distribution of primary radiation

$>$ Conditions of excitation geometry together with the construction features of the tube, the detector and the environment in which measurements are performed

$>$ The fundamental parameters of excitation elements and those of emission elements of the characteristic radiation

The fluorescence intensities are obtained from the processing of measured spectra. The energy distribution of the primary radiation is obtained from prepared models and is modified in accordance with the working conditions of the tube and its construction parameters (voltage, current, type and thickness of the anode, the type and thickness of the window, etc.). Fundamental parameters of excitation elements and those of emission of characteristic radiation elements are taken from tables or algorithms previously constructed for all the elements in the selected energy zone. Geometric conditions (distances, angles, environment, etc.) should be measured with precision for the system used in each case.

The main difficulty of using this method in the analysis of environmental samples, such as soil or plants, is related to the fact that we cannot measure the spectrum intensities of light elements $(\mathrm{O}, \mathrm{C}, \mathrm{N})$ that usually have high concentrations in the sample. The direct way to overcome this is to assume the presence of elements in an oxide form. In this way, the program takes into consideration the presence of oxygen and the mass reaches up to $100 \%$ [10], [12].

\subsection{FP Calibration and Analysis}

The XRF system should be calibrated before it can be used for routine quantitative analysis. There are two main parts in this calibration [11].

1. The first is the spectrometer calibration, which ensures that the X-ray peaks are found in the correct locations with respect to some known energy scale.

2. The second is the FP calibration of the individual elemental sensitivities, also known as calibration coefficients. However, this step may be ignored if a purely standardless analysis is used. 
A. Sinanaj, B. Papajani, The use of the fundamental parameters method..., RAD Conf. Proc., vol. 3, 2018, 25-31

In the case of our measurements, a soil sample whose spectrum showed evidence of $\mathrm{Ca}$ and Fe peaks was used as a calibration sample. The selected relation between the position (channel number) and the energy of peaks was linear and had this form:

$$
\mathrm{E}(\mathrm{keV})=0.2415(\mathrm{keV} / \mathrm{Ch}) * \mathrm{Ch}+0.015(\mathrm{keV})
$$

The next step is to calculate the factors responsible (sensitivity) for all the elements that we are interested in. This standardization process generates calibration coefficients that are further used by the main FP program. The easiest way to do calibration will be a standard with many elements.

The calibration process includes: (1) Measurement of standards in particular conditions of excitation. (2) Spectrum processing and obtaining intensities of lines of elements present in the standard. (3) Entering the concentrations of all the constituent elements of the standard into the program, including the concentration of the element of interest. (4) Calculation of the sensitivity coefficients from the program.

There are two ways of calibrating the sensitivity: a standard measurement of pure elements/compounds (special elements); and the measurement of a sample of a reference standard. As a standard for special elements, we used tablets prepared from pure compounds of elements and pure metal foil of some other elements. The Chinese reference standard GSS-4 (soil sample), the overall composition of which is precisely known, was used for the calibration of the standard value with multi-element.

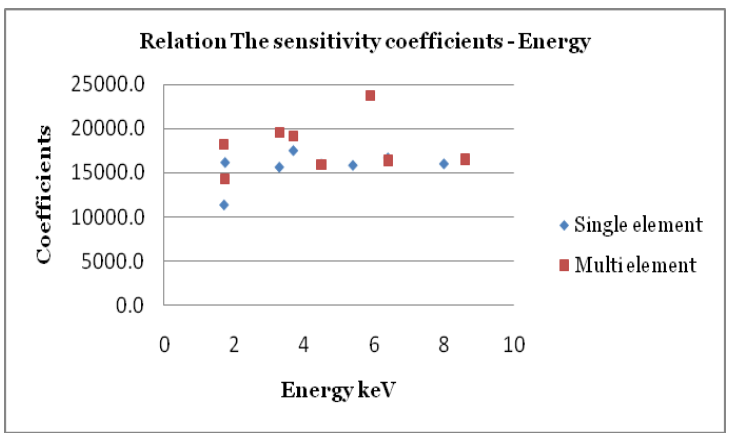

Figure 6 . The values of sensitivity coefficients from the energy
Calibration coefficient values, being more or less constant, at energy areas/zones that we are interested in, seem to represent a constant geometric system and depend on the measurement quality of the intensity (Al, Mn) and the accuracy of recognition of the concentrations. With all minor changes observed, these values were maintained in separate files and later were used to calculate specific concentrations.

\section{QUALITY CONTROL OF RESULTS}

Apart from the above-mentioned standards, a series of standards that were used for the quality control of the results were measured. The GSS and GSD Chinese standards were used in this way, representing soils and sediments of different types with a known composition. Standards are measured under the same system conditions while concentrations are calculated with 'standardless' and 'multi-element standard' methods that the FP program enables. In order to take into consideration the content of the light elements that do not appear in the spectrum $(\mathrm{O}, \mathrm{C}, \mathrm{N}$, etc.) in the 'standardless' method, the measured elements are treated as oxides and total content is normalized to $100 \%$. During the use of the 'multielement standard' method, the problem of lighter elements that do not appear in the spectrum is treated in two manners. The first manner uses fixed-content lightweight elements whose concentration is recognized and the measured elements are calculated as oxides, while the second manner uses the calculation of the measured elements as oxides, same as the 'standardless' method.

The results obtained for some of the standards are summarized in Table 1. The areas of concentrations, average deviations between the values measured using each method and the recommended values for all standards, the average deviation between logarithmic values measured using each method and the recommended algorithm values, and the limit values of $\Delta \log \mathrm{C}$ acceptable (refer to the criteria used for geochemical work that uses logarithmic deviation of concentrations as a criterion of the quality assessment of concentrations) for geochemical studies are presented for each element; (referring to Table 1) [11].

Table 1. Summarized results for each element and the average deviation

\begin{tabular}{|c|c|c|c|c|c|c|c|c|c|}
\hline \multirow[t]{2}{*}{ Elements } & \multicolumn{2}{|c|}{$\begin{array}{c}\text { Concentration } \\
\text { Area }\end{array}$} & \multicolumn{3}{|c|}{$\Delta(C)$ mean } & \multicolumn{3}{|c|}{$\Delta[\log (\mathrm{C})]$ mean } & \multirow{2}{*}{$\frac{\Delta \log C \text { limit }}{\%}$} \\
\hline & Min & Max & $\begin{array}{c}\text { Stand } \\
\text { less }\end{array}$ & $\begin{array}{l}\text { One stand } \\
\text { exact }\end{array}$ & $\begin{array}{l}\text { One stand } \\
\text { oxide }\end{array}$ & $\begin{array}{c}\text { Stand- } \\
\text { less }\end{array}$ & $\begin{array}{c}\text { One stand } \\
\text { exact }\end{array}$ & $\begin{array}{c}\text { One stand } \\
\text { oxide }\end{array}$ & \\
\hline Al & 5.18 & 14.7 & 1.48 & -0.25 & 1.50 & 0.11 & 0.09 & 0.10 & \pm 0.1 \\
\hline $\mathbf{S i}$ & 14.52 & 33.2 & -0.78 & 0.28 & 0.93 & -0.01 & 0.01 & 0.02 & \pm 0.1 \\
\hline $\mathbf{K}$ & 0.16 & 2.39 & 0.71 & 0.20 & 0.65 & 0.18 & 0.07 & 0.18 & \pm 0.2 \\
\hline $\mathbf{C a}$ & 0.065 & 5.62 & 0.22 & -0.12 & 0.19 & 0.06 & -0.06 & 0.06 & \pm 0.2 \\
\hline $\mathbf{T i}$ & 0.213 & 1.92 & 0.072 & -0.014 & 0.093 & 0.06 & 0.00 & 0.06 & \pm 0.2 \\
\hline Mn & 0.029 & 0.17 & 0.178 & 0.064 & 0.170 & 0.41 & 0.20 & 0.40 & \pm 0.2 \\
\hline $\mathbf{F e}$ & 1.33 & 12.4 & 0.60 & 0.11 & 0.62 & 0.06 & 0.02 & 0.06 & \pm 0.1 \\
\hline
\end{tabular}


A. Sinanaj, B. Papajani, The use of the fundamental parameters method..., RAD Conf. Proc., vol. 3, 2018, 25-31

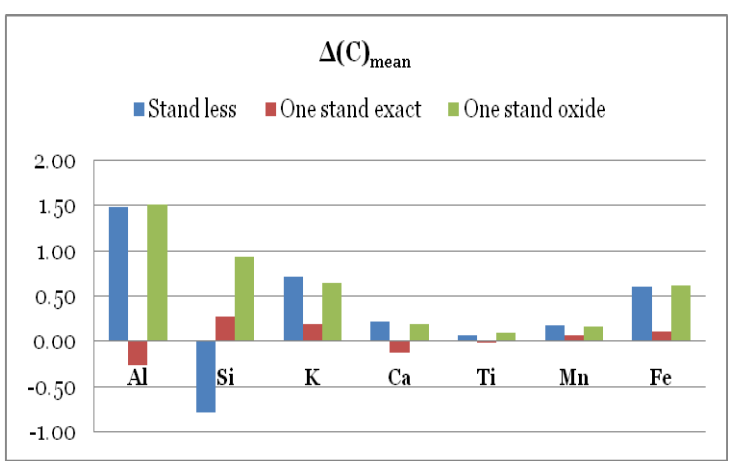

Figure 7. The comparison of the measured concentrations of elements with recommended values in different ways (the GSS Chinese standard)

The $y$-axis illustrate the average deviations of concentrations between the values measured using each method and the recommended values for all standards referring to Table 1.

The results presented show that the calculation of the concentration with the "Standardless" and "One multielement standard" methods, present approximate outcomes when the same method of assessing lighter elements (elements taken as oxides) is used. This proves that the geometry parameters included in the program are generally precise.

The results also show that taking into consideration the content of lighter elements that cannot be detected by an XRF device is important for obtaining accurate results with the FP-XRF method. Thus, the results obtained when the program includes information on the real content of lighter elements are generally in line with the values recommended. Average deviations are minimal, within the statistical limits, and are related mainly to the intensity of measured peaks and the manner of its calculation (small peaks and any instability of calibration), as in the case of $\mathrm{Al}$ and $\mathrm{Mn}$ which are small peaks close to the high peaks.

In case the content of lighter elements is not included and their presence is replaced with oxygen (elements as oxides), higher deviations between calculated concentrations and those recommended are observed, especially for elements with high concentrations (the concentration increases during normalization).

During the analysis of environmental samples, we do not have accurate data on the content of lighter elements and, therefore, we are obliged to store approximate data in the program, accepting a somewhat higher error. One way to improve the data obtained from FP-XRF in the future will be the adaptation of the results obtained for the standards with a straight line and the use of the line parameters to correct the results of unknown samples.

However, if we refer to the criteria used for geochemical work that uses logarithmic deviation of concentrations as a criterion of the quality assessment of concentrations, it is noted that all the methods used produce acceptable outcomes. In all cases, $\Delta \log C$ values are within the limits for these works, except Mn. We have to further improve our work for better results.

\section{CONCLUSION}

In this paper, in addition to the physical basis of the XRF method, special attention is paid to portable devices and the systems' method that uses fundamental parameters for calculating the concentrations in environmental samples analysis.

1. A portable XRF system consisting of a low-power X-radiation 'Mini X' tube, a cooling thermoelectric detector Si-PIN, the $\mathrm{PX}_{4}$ device, and the corresponding ADMCA PX4 and FP-XRF software are mounted and put into operation. Various experiments have led to the optimization of the geometry of the system, such as minimizing the distances, optimizing the angles, and assessing the beam spot in the position of the sample. Most of the data collected are used as initial data in the program that uses fundamental parameters for calculating the concentrations of elements in environmental samples.

2. The ADMCA program, which serves to control the working parameters of the system, obtain XRF spectra, and manipulate them, was put into operation and was used to measure a number of standard samples of soils and sediments, which were used to calculate the concentrations of elements with the Fundamental Parameter method (FP-XRF). After studying the structure and the opportunities provided by the FP-XRF program, the necessary calibration was carried out and used to calculate the concentrations of some of the standards used as samples.

3. Taking into consideration the content of the lighter elements that cannot be measured using the XRF system and which constitute a high percentage of the sample, a major problem affecting the quality of the results obtained by the FP method arises. The results presented show that the introduction of their content as approximate values partly solves that problem, enabling errors which can be acceptable to specific applications. Thus, calculating errors in the concentrations of sediment and soil samples can be acceptable for geochemical studies.

This research identified some aspects that can be used in the future to improve the results.

\section{REFERENCES}

1. R. Tertian, F. Claisse, Principles of Quantitative X-Ray Fluorescence Analyses, London, UK: Heyden, 1982.

2. I. Orlic, J. Makjanie, D. Raos, V. Valkovic, "A general way of solving matrix effect problems in elemental analysis by EDXRFS," $X$-Ray Spectrom., vol. 17, no. 4, pp. $139-143$, Aug. 1988.

DOI: $10.1002 /$ xrs.1300170405

3. E. P. Bertin, Introduction to X-ray Spectrometric Analyses, New York (NY), USA: Plenum Press, 1978. DOI: $10.1007 / 978-1-4899-2204-5$

4. W. Hanke, J. Wernisch, C. Pöhn, "Fluorescence yields, $\omega_{\mathrm{k}}(12 \leq \mathrm{Z} \leq 42)$ and $\omega_{\mathrm{LS}}(38 \leq \mathrm{Z} \leq 79)$, from $\mathrm{a}$ 
A. Sinanaj, B. Papajani, The use of the fundamental parameters method..., RAD Conf. Proc., vol. 3, 2018, 25-31

comparison of literature and experiments (SEM)," X-Ray Spectrom., vol. 14, no. 1, pp. 43 - 47, Jan. 1985. DOI: $10.1002 / \mathrm{xrs} .1300140110$

5. J. Sherman, "The theoretical derivation of fluorescent X-ray intensities from mixtures," Spectrochim. Acta, vol. 7, pp. $283-306,1955$.

DOI: 10.1016/0371-1951(55)80041-0

6. J.W. Criss, L.S. Birks, "Calculation methods for fluorescent x-ray spectrometry. Empirical coefficients versus fundamental parameters," Anal. Chem., vol. 40, no. 7, pp. 1080 - 1086, Jun. 1968. DOI: 10.1021/ac60263a023

7. T. Shiraiwa, N. Fujino, "Theoretical Calculation of Fluorescent X-Ray Intensities in Fluorescent X-Ray Spectrochemical Analysis," Jap. J. Appl. Phys., vol. 5, no. 10, pp. 886 - 899, Oct. 1966.

DOI: 10.1143/JJAP.5.886

8. W. H. McMaster, N. K. Delgrade, J. H. Mallet, J. H. Hubbel, Compilation of X-ray Cross Sections, Berkeley (CA), USA: University of California, 1969.

9. E. Vataj, "Perdorimi i metodes se Fluoreshences se rrezatimit X me dispersion energitik per studimin e objekteve rrej qelqi," disertacioni i M.Sc., Universiteti i Tiranës, Fakulteti i Shkencave Natyrore, Tirana, Albania, 2011. (E. Vataj, "Using the method of X-ray fluorescence with energy dispersive for the study of the glass objects," M.Sc. dissertation, University of Tirana, Faculty of Natural Sciences, Tirana, Albania. 2011.)

10. XRF FP Software guide v.3.3.o, Amptec, Bedford (MA), USA, 2016.

Retrieved from: https://www.physlab.org/wpcontent/uploads/2016/04/XRF-

FPSoftwareGuidev330.pdf;

Retrieved on: Jun. 13, 2018

11. XRS-FP Software Guide v.4.6.o, CrossRoads Scientific, El Granada (CA), USA, 2013.

Retrieved from: http://www.crossroadsscientific.com/C oncrete $5 /$ files/2013/3408/6160/XRS-

FP Software Guide v460.pdf;

Retrieved on: Jun. 13, 2018

12. XRF-FP Calibration and Auto-Run, Amptec, Bedford (MA), USA, 2018.

Retrieved from: http://compassweb.ts.infn.it/rich1/Ste fano/Amptek SW/Quantitative\%20Analysis\%20(XRFFP)/XRF-

FP\%20Analysis\%20with\%20Standards\%20and\%20Aut o-Mode.pdf;

Retrieved on: Jun. 13, 2018 